\title{
INFLUÊNCIA DO ATENDIMENTO FISIOTERAPÊUTICO HOME CARE EM IDOSOS PÓS COVID-19
}

\author{
INFLUENCE OF HOME CARE PHYSIOTHERAPY IN POST-COVID-ı ELDERLY
}

\author{
Ana Paula Lemes Menegatti ${ }^{2}$ \\ Rosangela Aparecida Brenneisen Fantin ${ }^{3}$ \\ Luciano Bernardes Júnior ${ }^{4}$
}

RESUMO: Introdução: As sequelas da covid - 19 requerem atenção especial em muitos casos, necessitando de um acompanhamento multidisciplinar para assegurar a qualidade de vida das pessoas afetadas. Objetivo: verificar a influência do atendimento fisioterapêutico em pacientes submetidos ao atendimento domiciliar. Métodos: estudo descritivo, transversal e quantitativo de campo. Os sujeitos da pesquisa foram quarenta e dois pacientes do gênero masculino e feminino, residentes da cidade de Balneário Camboriú que tiveram a covid-ı9, foram internados no Hospital Municipal Ruth Cardoso ou região que ficaram com algumas sequelas referente à doença e necessitaram de reabilitação de acordo com a avaliação fisioterapêutica, também participaram três fisioterapeutas atuantes no projeto do hospital. Resultados: $54,76 \%$ dos pacientes classificam sua saúde mental como "boa ou excelente" comparado a antes do COVID-ı9; a saúde física foi classificada como "boa ou excelente" por $35,71 \%$ dos pacientes e como "razoável" por 33,33\%; todos os participantes avaliaram como "excelente" as questões relacionadas à interação paciente-fisioterapeuta; I0o\% responderam que indicariam a fisioterapia a outras pessoas; quanto a importância do acompanhamento fisioterapêutico resultados indicaram um percentual alto de 95,24\%; na saúde mental 95,24\% relataram com "muito ou bastante" a influência dos atendimentos fisioterapêuticos; 100\% demonstraram contentamento no atendimento domiciliar; 100\% relataram que se sentiram "bem ou excelente" após o atendimento fisioterapêutico. Conclusão: Houve resposta positiva na qualidade de vida de uma forma geral, na respiração, mentalmente e fisicamente.

Palavras-chave: Covid-19. Fisioterapia. Idosos.

ABSTRACT: Introduction: The sequelae of covid - 19 , special attention in many cases, requiring multidisciplinary monitoring to ensure the quality of life of affected people. Objective: to verify the influence of physical therapy care in patients undergoing home care. Methods: descriptive, crosssectional and quantitative field study. The research subjects were forty-two male and female patients, residents of the city of Balneário Camboriú who had covid-r9, were admitted to the Ruth Cardoso

\footnotetext{
${ }^{1}$ Trabalho apresentado à disciplina de Trabalho de Conclusão de Curso II, do Curso de Fisioterapia do Centro Universitário Avantis - UNIAVAN, 2020/or.

2 Acadêmica do curso de Fisioterapia. E-mail: paullann@hotmail.com.

3 Acadêmica do curso de Fisioterapia. E-mail: rbrenneisen@hotmail.com

${ }^{4}$ Especialista. E-mail: Luciano.bernardes@uniavan.edu.br.
} 
Municipal Hospital or region, who had some sequelae related to the disease and required rehabilitation of According to the physical therapy evaluation, three physical therapists working in the hospital project also participated. Results: $54.76 \%$ of patients classify their mental health as "good or excellent" compared to before COVID-I9; physical health was classified as "good or excellent" by $35.71 \%$ of patients and as "fair" by 33.33\%; all participants rated as "excellent" the issues related to the patient-physiotherapist interaction; I0o\% answered that they would recommend physiotherapy to other people; regarding the importance of physical therapy follow-up, results indicated a high percentage of $95.24 \%$; in mental health, 95.24\% reported with "a lot or a lot" the influence of physical therapy care; $100 \%$ demonstrated contentment in home care; Ioo\% reported that they felt "good or excellent" after physical therapy care. Conclusion: There was a positive response in terms of quality of life in general, in breathing, mentally and physically.

Keywords: Covid -I9. Physiotherapy. Elderly.

\section{INTRODUÇÃO}

A pandemia global causada por covid-rg afetou milhares de pessoas e causou muitas mortes, desses afetados que foram curados, muitos ficaram com sequelas associadas à doença. Estudos mostram que as implicações vão além do comprometimento do sistema respiratório, prejudicando diversos sistemas, tais como distúrbios neurológicos, gastrointestinais e musculoesqueléticos (NICOLA et al, 2020). Esse grupo de vírus é responsável por causar síndromes respiratórias agudas que podem variar de sintomas leves a condições graves, podendo levar a internação hospitalar, necessidade de ventilação e significativa taxa de mortalidade (ROTHAN et al, 2020).

Baptista et al (2020) relataram que as complicações mais frequentemente observadas foram a sepse, insuficiência respiratória e choque séptico, também relatam que as chances de morte hospitalar foram maiores em pacientes com diabetes ou doença coronariana, a idade também foi associada à morte. Silva et al (2020) especificam que as maiores complicações deixadas pela doença são vistas nos pacientes que dependeram de internações em Unidades de Terapia Intensiva (UTI), pois a permanência prolongada nos leitos colabora de forma negativa na qualidade de vida desses pacientes.

No envolvimento da recuperação física do paciente com covid-rg atua uma equipe multiprofissional com destaque ao fisioterapeuta, não por tratar a doença em si, mas por ser agente indispensável na prevenção e reabilitação das deficiências respiratórias e nas limitações funcionais da atividade de vida diária por ela ocasionadas (BISPO JÚNIOR, 2020). Avila et al (2020) denotam que a abordagem terapêutica deve ser individualizada levando em consideração os perfis, as comorbidades, a qualidade da assistência, o tempo de internação e o uso da ventilação mecânica invasiva. 
É essencial a atuação do fisioterapeuta desde a fase mais precoce da doença. Seu acompanhamento deve ser viável com a situação do paciente, tais como o telemonitoramento à distância em casos de isolamento, no ambiente hospitalar nos casos que necessite de hospitalização, visando promover recuperação funcional e acelerar o processo de alta e indispensavelmente na recuperação pós COVID-I9. Durante a fase aguda da doença o papel do fisioterapeuta não se restringe ao sistema respiratório, é indispensável sua atuação na condução de exercícios e mobilizações que minimizarão significativamente os déficits musculoesqueléticos decorrentes do imobilismo prolongado (SILVA et al, 2020).

O presente estudo demonstra a influência da fisioterapia no tratamento pós covid-ı́ em pacientes idosos, acompanhados por fisioterapeutas na modalidade home care, através do projeto de extensão do Centro Municipal de Atendimento e Tratamento Covid 19, programa de Fisioterapia Domiciliar PósCovid 19, realizado no município de Balneário Camboriú - SC, o projeto atende pacientes idosos que tiveram a covid 19, ficaram com alguma sequela e necessitam de reabilitação de acordo com a avaliação dos fisioterapeutas que atuam nesse projeto. Por se tratar de uma doença recente ainda não se sabe em longo prazo quais são as consequências físicas, por isso há necessidade de um acompanhamento para melhoria dos sintomas atuais e prevenção de efeitos secundários.

O objetivo foi verificar a influência do atendimento fisioterapêutico em pacientes adultos pós covid-ı, submetidos a atendimento domiciliar, verificando a qualidade de vida, avaliando a satisfação com o tratamento, analisando a importância do acompanhamento fisioterapêutico, verificando se houve melhoras após o tratamento e descrevendo a experiência do fisioterapeuta nas visitas home care.

\section{MÉTODOS}

Foram selecionados para o presente estudo quarenta e dois pacientes idosos pós a covid I9, de ambos os sexos, também três fisioterapeutas, ambos participantes do Programa Fisioterapia Domiciliar a Idosos Pós-Covid I9, um projeto de extensão do Centro Municipal de Atendimento e Tratamento Covid I9 do município de Balneário Camboriú.

Inicialmente para a realização da pesquisa foram coletados os dados dos pacientes por meio das fichas de avaliação já elaboradas pelos fisioterapeutas que atuam no projeto, como dados pessoais, contato telefônico, queixa principal, sinais e sintomas, morbidades prévias e número de atendimentos fisioterapêuticos realizados. 
Foi realizada a coleta por meio de questionários elaborados com perguntas objetivas para os pacientes e discursivas para os fisioterapeutas, os dados foram coletados em um dia útil da semana, no período diurno. O questionário dos pacientes foi aplicado por meio de ligações telefônicas, com o tempo de aplicação necessário para os participantes responderem a todas as perguntas elaboradas, a realizar-se dentro de uma semana todas as ligações. O questionário dos fisioterapeutas foi elaborado pelo Google Forms e encaminhado à coordenadora responsável do projeto para que fosse encaminhado via whatsapp dos fisioterapeutas. Os participantes foram convidados a participar da pesquisa no momento que antecedeu a aplicação do questionário, assim sendo informados sobre os objetivos do questionário, juntamente com as informações de como a coleta de dados e a pesquisa foram desenvolvidos, sendo que a mesma somente foi realizada com o termo de consentimento livre e esclarecido (TCLE) devidamente assinado pelos pacientes e fisioterapeutas. Os termos foram encaminhados pelos pacientes através dos fisioterapeutas do projeto, por via whatsapp e também por e-mail.

Os dados quantitativos foram armazenados e analisados no programa Statistical Package for the Social Science (SPSS) versão 24.o. Foi realizada análise descritiva por meio de frequência, média e desvio padrão. Os dados qualitativos foram transcritos para um arquivo do Microsoft Word para facilitar as análises.

\section{I APROVAÇÃO DO PROTOCOLO E CONSIDERAÇÕES ÉTICAS}

Este estudo foi aprovado pelo Comitê de Ética em Pesquisa sob parecer número 4.6r8.952. Realizado conforme a resolução 466/22.

Todos os participantes assinaram o Termo de Consentimento Livre e Esclarecido (TCLE), que visa o respeito devido à dignidade humana. $\mathrm{O}$ anonimato dos participantes da pesquisa foi resguardado, a partir do posicionamento ético dos pesquisadores a fim de garantir a manutenção dos cuidados éticos da pesquisa.

\subsection{CARACTERIZAÇÃO DA PESQUISA}

Esta pesquisa caracteriza-se por ser descritiva, transversal e quali-quantitativa.

\section{3 SELEÇÃO DOS PARTICIPANTES DO ESTUDO}

Dentre os participantes que responderam os questionários foram recrutados somente os fisioterapeutas colaboradores do projeto do hospital e somente os idosos que tiveram atendimentos fisioterapêuticos domiciliares pós covid 19 com no mínimo três sessões de reabilitação, que pudessem 
responder todo o questionário por meio de ligação telefônica e que tivessem assinado o termo de consentimento livre e esclarecido o qual foi entregue pelos fisioterapeutas do projeto por via whatsapp e/ou e-mail. O contato telefônico dos participantes foi obtido com o cadastro do projeto sob aceite da coordenação do Hospital Municipal Ruth Cardoso com o termo de autorização para utilização documental e dados clínicos em pesquisa devidamente assinados.

Os critérios de inclusão foram com bases sobre a ficha cadastral do participante do projeto, obtido após o aceite da autorização do mesmo.

Os critérios de exclusão foram idosos que não assinaram os termos de consentimento livre e esclarecido, que possuíam algum distúrbio cognitivo e os que de alguma forma não conseguiram responder todo o questionário por meio de ligação telefônica. Foram selecionados 50 pacientes, mas somente 42 atenderam as ligações telefônicas e responderam o questionário. Dos fisioterapeutas foram selecionados 4 , porém apenas 3 responderam o questionário por completo.

\subsection{PROCEDIMENTOS}

Inicialmente para a realização da pesquisa foram coletados os dados dos pacientes, a queixa principal, morbidades prévias, sinais e sintomas e o número de atendimentos fisioterapêuticos realizados com cada idoso por meio das fichas de avaliação já elaboradas pelos fisioterapeutas atuantes do projeto.

Foi realizada a coleta por meio de questionários elaborados com perguntas objetivas para os pacientes contendo quinze perguntas relacionadas à saúde mental, saúde física e sobre a intervenção da fisioterapia. Para os fisioterapeutas foi elaborado um questionário discursivo contendo dezessete perguntas divididas em três categorias: referente à forma de atendimento, quanto à formação do profissional e referente ao projeto. Os dados foram coletados em um dia útil da semana, no período diurno.

O questionário dos pacientes foi aplicado por meio de ligações telefônicas, com o tempo de aplicação necessário para os participantes responderem a todas as perguntas elaboradas, a realizar-se dentro de uma semana todas as ligações.

O questionário dos fisioterapeutas foi elaborado pelo Google Forms e encaminhado à coordenadora responsável do projeto para ser encaminhado via whatsapp dos fisioterapeutas.

Participaram deste estudo 42 pacientes, sendo 2I do sexo feminino e 2I do sexo masculino, tendo estes idade entre 60 anos e 79 anos, peso de $55 \mathrm{~kg}$ a $124 \mathrm{~kg}$ e altura de $1,54 \mathrm{~m}$ a $1,87 \mathrm{~m}$. 
A seguir, serão apresentados os gráficos relacionados ao estudo em questão.

\section{RESULTADOS}

Os gráficos I a 5 representam os dados referentes à qualidade de vida dos indivíduos acompanhados pelo serviço de fisioterapia.

Observa-se que $54,76 \%$ dos pacientes classificam sua saúde mental como "boa ou excelente" comparado

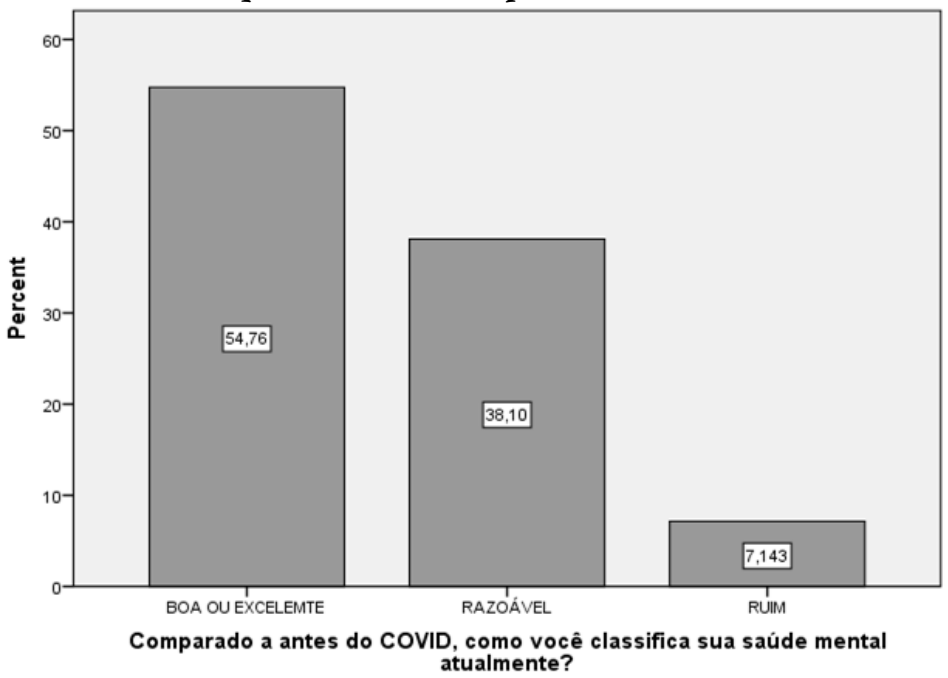

a antes do COVID-ı9 (gráfico I).

Em relação à preocupação atual com a saúde, 52,38\% dos participantes relataram que se preocupam “muito ou bastante" (gráfico 2). Já quando questionados sobre o quanto o COVID-I9 afetou sua vida, $78,57 \%$ se sentiram "muito ou bastante afetados" (gráfico 3 ).

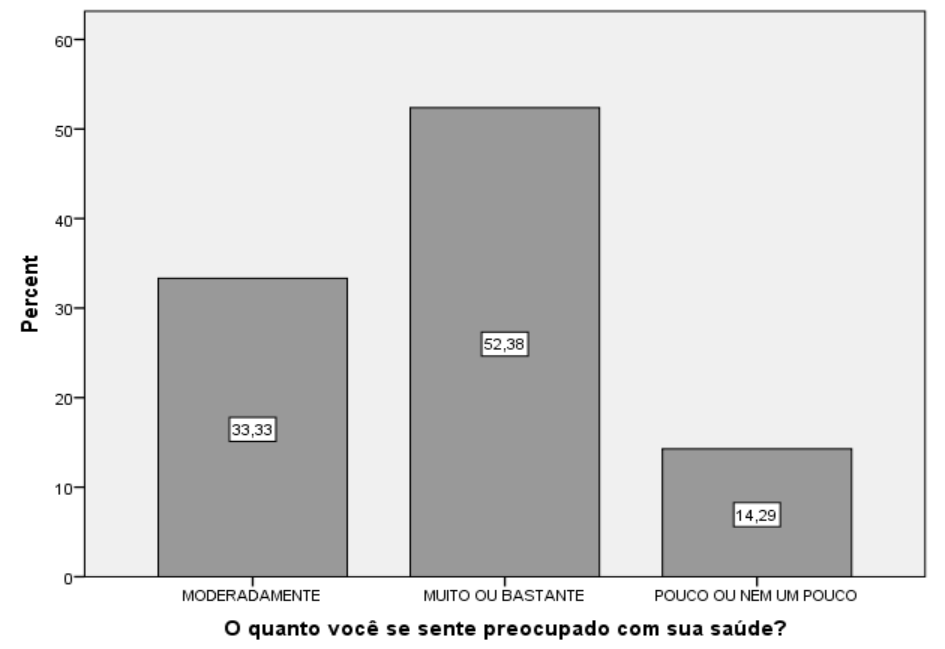


Gráfico 2. Classificação da saúde mental pelo relato dos participantes. Fonte: dados primários.

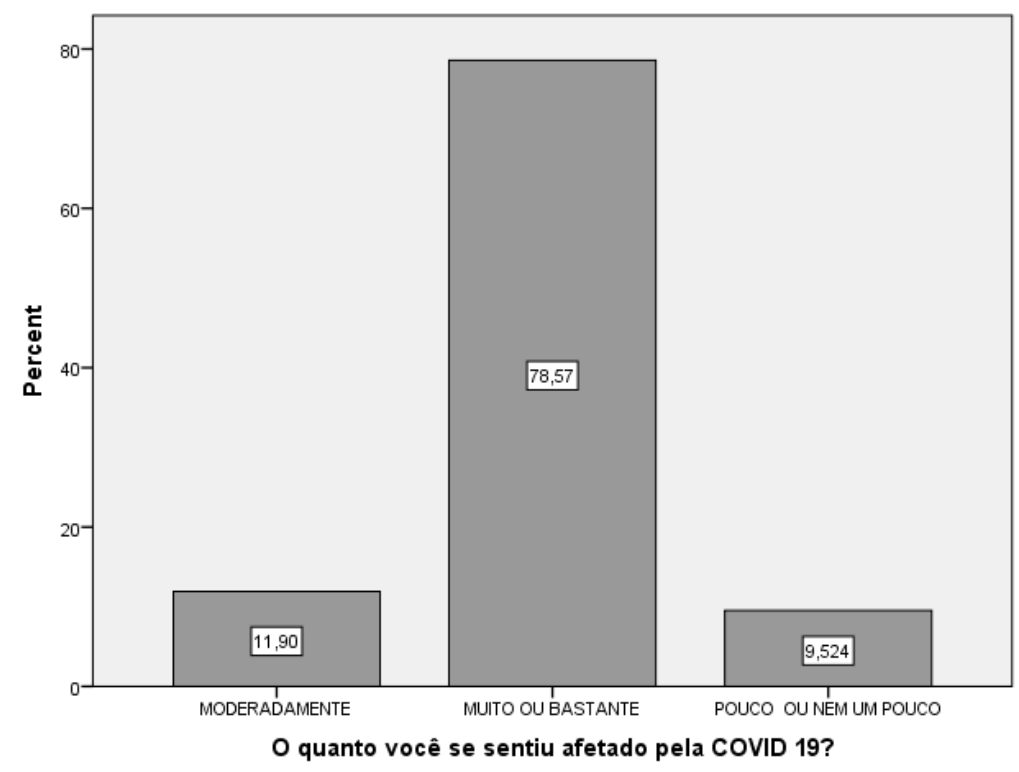

Gráfico 3. Classificação da saúde mental pelo relato dos participantes. Fonte: dados primários.

A saúde física foi classificada como "boa ou excelente" por 35,71\% dos pacientes e como "razoável" por 33,33\% (gráfico 4). Além disso, 47,62\% (gráfico 5) relataram que a COVID-ı limitou “muito" suas atividades de vida diária.

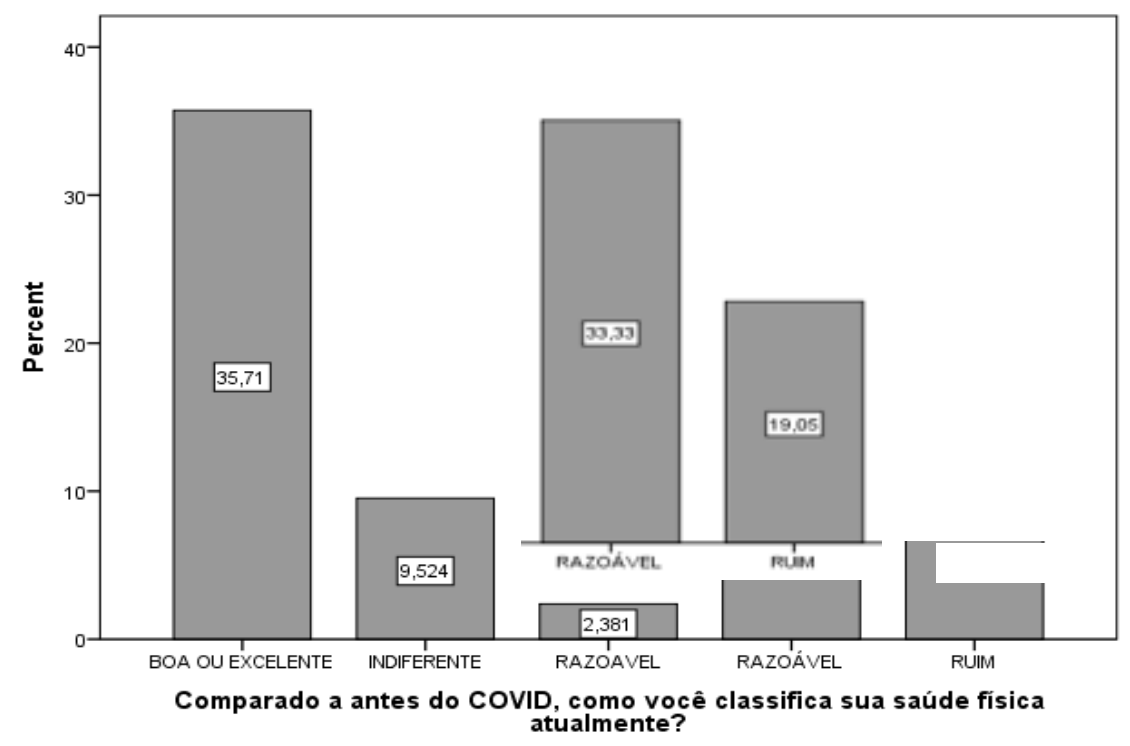


Gráfico 4. Classificação da saúde física pelo relato dos participantes. Fonte: dados primários.

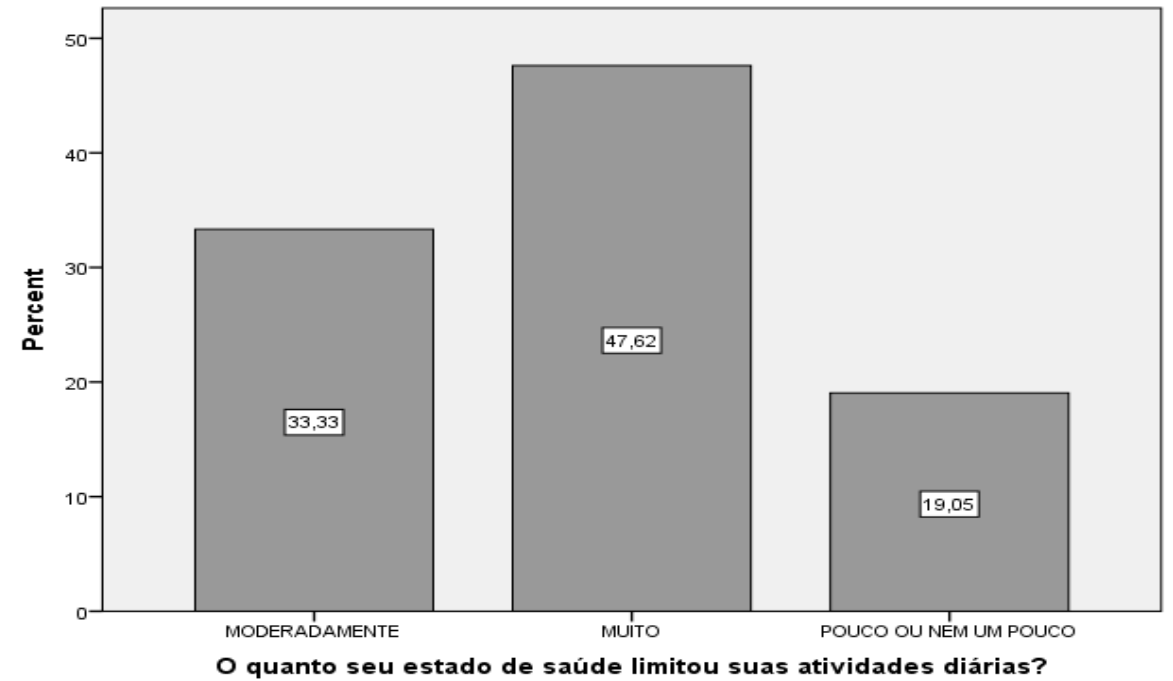

Gráfico 5. Classificação da saúde física pelo relato dos participantes. Fonte: dados primários.

Quando questionados quanto ao atendimento da fisioterapia, todos os participantes (Ioo\%) avaliaram como excelente as questões relacionadas à interação paciente-fisioterapeuta. Ainda, todos os participantes manifestam satisfação, o que nos leva a um resultado de $100 \%$ em relação aos atributos e atenção oferecida aos pacientes, pois os mesmos sentem-se favorecidos pelo tratamento recebido e indicariam a fisioterapia a outros pacientes.

A importância do acompanhamento fisioterapêutico foi destacada nos resultados que indicam um percentual alto de $95,24 \%$ com resposta referente a "muito ou bastante". Esse fato se justifica pelo fisioterapeuta estabelecer uma relação com o paciente revelando interesse na melhora do mesmo, esclarecendo suas dúvidas e dando explicações referentes ao que estava sendo realizado no tratamento. Motivando o paciente durante o tratamento a alcançar a melhora no seu quadro.

Referente a classificação da influência na qualidade da respiração pelo relato dos participantes após os atendimentos, pudemos obter resultados positivos com 95,24\%, relatando com "MUITO OU BASTANTE” a influência dos atendimentos na qualidade da respiração.

Quando questionados sobre os atendimentos serem realizados no próprio domicilio, os pacientes relataram a classificação de ıoo\%, os resultados indicaram contentamento dos usuários com a assistência domiciliar. Todos os pacientes avaliaram o atendimento relatando que se sentiram 
“BEM/EXCELENTE” após o atendimento da fisioterapia, devido à atenção recebida, ética, eficiência e interesse demonstrado pelos profissionais e pela equipe responsável.

\section{EXPERIÊNCIA DO FISIOTERAPEUTA NO ATENDIMENTO HOME CARE DO PACIENTE PÓS COVID rg:}

O questionário aplicado aos fisioterapeutas buscou entender como o projeto funciona aos olhos do profissional, bem como compreender quais métodos utilizados durante as visitas, incluindo aspectos referentes à forma que eram acolhidos pelo paciente.

Para compreensão da forma que ocorrem os atendimentos, pode-se destacar a fala de um dos fisioterapeutas do projeto: "Na avaliação buscamos identificar como a pessoa se manifesta no meio em que está inserida, do ponto de vista funcional, emocional e social, para assim direcionar a terapêutica adequada, de forma personalizada. Pontualmente, abordamos informações contendo os dados pessoais, a história da moléstia pregressa e atual, qual seria a queixa principal, se essa pessoa possui comorbidades, se faz uso medicamentos e se possui fatores de risco. Além do mais, aplicamos os questionários Barthel e SF-36 para reconhecer de que forma o indivíduo executa suas atividades de vida diária (AVD's) e como está a sua qualidade de vida, ainda, aplicamos as escalas MRCdispneia e IMS para avaliar a limitação imposta pela dispneia e sua mobilidade”.

Quanto ao perfil do paciente atendido pelo projeto, outro fisioterapeuta aborda a singularidade de cada indivíduo como fator importante no tratamento: "Em vista dos diferentes graus de complicações prévias e adquiridas, tempo de internação, idade, fatores de risco, comorbidades, entre outros, a resposta é bem variada. Mas de certa forma a cada intervenção observamos alguma melhora. Tivemos pacientes que na primeira semana receberam alta e aqueles que permanecem conosco até os dias atuais, numericamente é a minoria. Fazendo uma média do início do projeto e tempo de alta, temos o valor de 58 dias, ou seja, praticamente 2 meses".

No que tange à formação dos fisioterapeutas, o tempo de experiência profissional relatado foi entre 3 a 16,7 anos, todos declaram possuir experiência em fisioterapia respiratória e domiciliar.

Questionados sobre desafios da Pandemia, relataram:

"Grande necessidade de adaptação individual e coletiva".

"Conscientização da população".

"Manter a saúde mental e física e acreditar que tudo vai passar".

Referente ao projeto, os fisioterapeutas informaram que o deslocamento até à casa dos pacientes era seguido por um itinerário realizado pelo secretário do projeto e a Secretaria da Saúde disponibilizava 
o motorista e o veículo. Durante o período da pesquisa os atendimentos de 8 a I4 pacientes eram realizados diariamente.

Questionados sobre os equipamentos para auxiliar nas condutas obtivemos a seguinte resposta de um dos fisioterapeutas: "Sim, é disponibilizado todos os equipamentos da avaliação (manovacuômetro, peak flow, clip nasal, filtro HME, dinamômetro manual, step, caneleira), os EPI's (touca, jaleco descartável, óculos, máscara n95, luva descartável), de descarte de lixo contaminado e higienização (saco de lixo contaminado, álcool) e equipamentos como ciclo ergômetro, miniband, theraband, halter, caneleira, cones, escada de agilidade, entre outros”. Todos disponibilizados pelo hospital.

Questionados sobre os equipamentos para auxiliar nas condutas obtivemos a seguinte resposta de um dos fisioterapeutas: "Sim, é disponibilizado todos os equipamentos da avaliação (manovacuômetro, peak flow, clip nasal, filtro HME, dinamômetro manual, step, caneleira), os EPI's (touca, jaleco descartável, óculos, máscara n95, luva descartável), de descarte de lixo contaminado e higienização (saco de lixo contaminado, álcool) e equipamentos como ciclo ergômetro, miniband, theraband, halter, caneleira, cones, escada de agilidade, entre outros". "Todos disponibilizados pelo hospital”.

Referente à opinião dos fisioterapeutas sobre o projeto relataram as seguintes respostas: relato I“Acho este projeto fantástico, é uma honra poder participar e contribuir para a execução e continuidade do mesmo. Ver esse tipo de ação no SUS, com agilidade e resolutibilidade me enche de felicidade. Estamos aqui falando de um projeto para Idosos pós COVID-ı, agora vamos ampliar um pouco mais, há muitas pessoas que não possuem idade igual ou superior a 60 anos e se beneficiariam muito, bem como aqueles que não se relacionam com o COVID mas que também precisam de atenção de cuidado fisioterapêutico domiciliar." Relato 2-O projeto tem sido fundamental para devolver a funcionalidade aos idosos pós covid, visto que grande parte não teria acesso a esse serviço se ele não fosse oferecido pelo SUS e a domicílio. Projeto parecido como este, poderia atuar também em reabilitação precoce pós alta hospitalar de pacientes ortopédicos e neurológicos".

Sobre o questionamento se o atendimento home care faz o suporte necessário para os pacientes e desafoga o sistema e se os objetivos pessoais foram alcançados relataram que, como o público é bem restrito conseguem dar conta da demanda, e que não só desafoga o sistema como potencializa o resultado com a intervenção precoce, e relataram que os objetivos pessoais com certeza foram alcançados. 


\section{DISCUSSÃO}

Dados de vários estudos demonstram que a Covid-ı́ é um risco particular para as pessoas idosas, principalmente para aquelas que possuem multimorbidades, a taxa de mortalidade é significativamente maior que nas crianças e adultos com idade menor que 60 anos (Costa et al, 2020). No resultado do nosso estudo sobre o quanto a covid afetou a vida de uma forma geral, $78,57 \%$ se sentiram muito ou bastante afetados tanto na saúde mental como física, um dado notável é que a maioria dos idosos possuíam muitas comorbidades em destaque a hipertensão arterial e diabetes e faziam uso de vários medicamentos durante o dia. Ferreira et al (2020) discorrem sobre a importância de se manter ativo em tempos de isolamento social onde a população se mantém reclusa adotando uma rotina sedentária o que favorece o surgimento das comorbidades.

Em uma revisão de literatura Silva et al (202I) identificaram que a reabilitação respiratória logo após a alta hospitalar resultou em respostas positivas quanto à respiração e a qualidade de vida, ressalta ainda que a intervenção precoce deve começar dentro da UTI e logo após a alta a intervenção fisioterapêutica deve ser focada nas funcionalidades e dificuldades de realização das atividades de vida

diária. Santana et al (202I) informam que devido o comprometimento multissistêmico causada pela Covid-ı9 é ideal que uma equipe multiprofissional conduza a reabilitação.

Os pacientes que responderam essa pesquisa tiveram um acompanhamento fisioterapêutico precoce desde a internação no hospital até o momento de alta hospitalar que deveriam se cadastrar no projeto de reabilitação para continuarem a ter o suporte em seus domicílios. Como podemos notar esse acompanhamento é de extrema importância tanto para o hospital para a monitoração da saúde desses idosos como para os próprios beneficiados com esse projeto, cujo resultado é notável nos resultados quantitativos acima, que apresentaram valores de $90 \%$ a 100\% de repostas positivas sobre a influência da fisioterapia quanto à qualidade da respiração, saúde mental e bem estar geral.

Quanto aos atendimentos serem realizados em seus domicílios I0o\% se sentiram muito confortáveis tanto por não dependerem de deslocamentos até uma unidade de reabilitação devido a debilidade ocasionada pela covid, como por manterem o isolamento em tempo de pandemia. Pereira e Gessinger (2014) relatam que os idosos podem ter agravamento em seu estado de saúde ocasionado pela dificuldade no acesso ao serviço de saúde, devido muitos possuírem doenças crônicas limitantes ou até mesmo estarem limitados ao leito o que demonstra a importância da atenção domiciliar. 
Durante a entrevista muitos relataram que já conheciam a fisioterapia, mas de uma visão totalmente diferente, não tinham todo o conhecimento de que a fisioterapia vai muito além da reabilitação ortopédica, abrangendo todo o contexto tanto fisicamente quanto psicologicamente. Gonçalves e Alves (2021) analisaram a valorização da fisioterapia respiratória frente a covid 19 e destacam que sim, houve um grande reconhecimento dessa área tanto pela necessidade da atuação em uma equipe multidisciplinar quanto pela sociedade em conhecer o papel da fisioterapia. Também descreveram que os fisioterapeutas esclareceram todas suas dúvidas e que a interação paciente-fisioterapeuta foi excelente, se sentindo favorecidos com o tratamento.

Referente ao questionário respondido pelos fisioterapeutas é notável como se sentem lisonjeados de participarem de um programa tão importante para a reabilitação dos idosos pós a covid I9, disponibilizado por um hospital público, onde tem total apoio desde ao deslocamento com suporte de carro e motorista disponibilizado até aos equipamentos necessários para a reabilitação desses idosos, onde eles têm o contato desde a internação até seus domicílios e à alta da fisioterapia, o que na maioria dos casos em hospitais que não contam com um serviço de acompanhamento desses pacientes os fisioterapeutas não conseguem acompanhar a evolução do quadro de saúde, saber se progrediram ou regrediram após a alta hospitalar.

Pinheiro et al (2017) em seu estudo concluíram que a importância de um acompanhamento pós alta hospitalar possibilita o monitoramento da evolução dos pacientes, também viável a identificar situações de risco, prevenir complicações e reinternações. Camargo et al (2016) também reforça a necessidade do acompanhamento e orientações pós alta hospitalar por uma equipe multiprofissional, relata que a carência dessas atividades gera falta de conhecimento e preparo nos cuidados prestados, possíveis complicações secundárias e novas reinternações.

Houve algumas limitações em nosso estudo em relação ao número de participantes e a metodologia de coleta de dados. Devido a coleta com os pacientes ser realizada por meio de ligações telefônicas, grande parte não atendeu as chamadas. Em muitos casos, os contatos estavam incorretos ou alegavam falta de tempo para responder as perguntas.

\section{CONCLUSÃO}

O questionário aplicado na entrevista com os pacientes foi capaz de demonstrar que a influência da fisioterapia na reabilitação precoce pós a covid I9 propicia qualidade de vida de forma geral, tanto na 
saúde mental, física e respiratória. Também demonstrou o contentamento dos pacientes em serem atendidos em seus próprios domicílios e terem esse acompanhamento de forma gratuita.

O questionário aplicado com os fisioterapeutas nos demonstrou que ter o apoio em um projeto de reabilitação tão importante como este é gratificante em acompanhar a evolução de um paciente que antes estava entubado como em alguns casos e com risco de morte, evoluir para uma alta hospitalar e ser acompanhado até à sua alta fisioterapêutica em seu próprio domicílio. Assim gera questionamentos acerca de que projetos como este deveriam existir para todos os pacientes que necessitam de acompanhamento após a alta hospitalar, seja pós covid, neurológicos, ortopédicos entre outros.

\section{REFERÊNCIAS.}

AVILA, Paulo Eduardo Santos; PEREIRA, Raphael do Nascimento; TORRES, Daniel da Costa. Guia de orientações fisioterapêuticas na assistência ao paciente pós COVID-19. Belém: UFPA, FFTO, Curso de Fisioterapia, 2020. Disponível em: https://livroaberto.ufpa.br/jspui/handle/prefix/833. Acesso em: 30 de jun de 202I.

BARBOSA BAPTISTA, A.; VIEIRA FERNANDES, L. Covid-ı́, análise das estratégias de prevenção, cuidados e complicações sintomáticas. DESAFIOS - Revista Interdisciplinar da Universidade Federal do Tocantins, v. 7, n. Especial-3, p. 38-47, 22 abr. 2020. Disponível em: $<$ https://sistemas.uft.edu.br/periodicos/index.php/desafios/article/view/8779> Acesso em 30 de jun de 2021.

\section{BISPO JÚNIOR, J.P. Fisioterapia e saúde coletiva: Desafios e novas responsabilidades profissionais.}

Cienc Saude Coletiva. 2010. Disponível em:https://www.scielo.br/scielo._arttext\&pid=Si413-. Acesso em ro de set de 2020 .

\section{CAMARGO, Patrícia Faria; ANDRÉ, Larissa Delgado; LAMARI, Neuseli Marino. Orientações em} saúde no processo de alta hospitalar em usuários reinternados do sistema único de saúde. Arquivos de Ciências da Saúde, [S.1.], v. 23, n. 3, p. 38-43, nov. 2016. ISSN 2318-3691. Disponível em: 〈https://www.cienciasdasaude.famerp.br/index.php/racs/article/view/335〉. Acesso em: 30 jun. 202I. doi: https://doi.org/10.17696/2318-3691.23.3.2016.335. 
COSTA, F. A. et al. COVID - I9: seus impactos clínicos e psicológicos na população idosa. Braz. J. of Develop., Curitiba, v. 6, n. 7, p. 498II-49824 jul . 2020. Disponível em: <https://www.brazilianjournals.com/index.php/BRJD/article/view/13704/Ir475. Acesso em i9 de jun de 202I

FERREIRA, M. J. et al. Vida Fisicamente Ativa como Medida de Enfrentamento ao COVID-19. Arq Bras Cardiol. 2020; II4(4):601-602. Disponível em: 〈https://www.scielo.br/j/abc/a/9kVQrK5VcGsXzg3xLhyWTNL/?format=pdf\&lang=pt〉. Acesso em: 26 de jun de 2021.

GONÇALVES, E. H., ALVES, L. F. Valoriação da Fisioterapia Respiratória frente a Covid-ı. Anais do Congresso Nacional Universidade, EAD e Software Livre, vol.r n 12, 2021. Disponível em : 〈http://www.periodicos.letras.ufmg.br/index.php/ueadsl/article/view/r8270〉. Acesso em 26 de jun de 2021.

NICOLA M., ALSAFI Z., SOHRABI C., KERWAN A., AL-JABIR A., IOSIFIDIS C., et al. The socio-economicimplicationsofthecoronavirusand COVID-19 pandemic: a review. Int J Surg. 2020. Disponível em: https://www.sciencedirect.com/science/article/abs/pii/Si743919120303162. Acesso em: Io de out de 2020 .

PEREIRA, B.M. GESSINGER, C.F. Visão da equipe multidisciplinar sobre a atuação da fisioterapia em um programa de atendimento domiciliar público.O Mundo da Saúde, São Paulo - 2014;38(2):210-218. Disponível em:

$\langle$ https://www.saocamilo-sp.br/pdf/mundo_saude/I55562/Aio.pdf $>$. Acesso em i9 de jun de 202I.

PINHEIRO. F. et al. Acompanhamento por telefone no pós alta hospitalar de idosos. Revista Enfermagem Atual, 2017. Disponível em: 〈http://revistaenfermagematual.com.br/index.php/revista/article/download/284/175〉 Acesso em 29 de jun de 2021. 
ROTHAN H.A., BYRAREDDY S.N. The Epidemiology and pathogenesis of coronavirus disease (COVID-19) outbreak. J Autoimmun. 2020. Disponível em:

https://www.sciencedirect.com/science/article/pii/So896841120300469. Acesso em ro de out de 2020.

SANTANA, A. V. et al. Reabilitação Pulmonar Pós Covid-19. Editorial, J. bras. pneumol. 47(I), 2021. Disponível em: 〈https://www.scielo.br/j/jbpneu/a/nXKFpxSjzHpgw8893y77c6L/?lang=pt >Acesso em 29 de jun de 202I.

SILVA, L. C. O. et al. Sequelas e Reabilitação Pós-Covid 19: Revisão de Literatura. Revista das Ciências da Saúde e Ciências aplicadas do Oeste Baiano-Higia. 202I; 6(I):I69-I84. Disponível em: <file:///tmp/mozilla_usuarioo/637-2114-I-PB.pdf. Acesso em ig de jun de 2021.

SILVA, R. M. V., SOUZA, A. V. C. Fase crônica da COVID-rg: desafios do fisioterapeuta diante das disfunções musculoesqueléticas. Fisioter. Mov. Vol.33, 2020. Disponível em:

http://www.scielo.br/scielo.php?script=sci_arttext\&pid=So103-51502020000100101\&lng=en. Acesso em 23 de set de 2020 . 